\section{P121 DO GUIDELINES HELP PROMOTE SEXUAL HEALTH SCREENING (SHS) AND DETECT SEXUALLY TRANSMITTED INFECTIONS (STIS) IN OLDER ADULTS WITH HIV?}

doi:10.1136/sextrans-2012-050601c.121

A Baugh, R Taylor, ${ }^{*}$ L Bellingham, I Ahmed. Nottingham University Hospitals NHS Trust, Nottingham, UK

Background Adults over 50 with HIV comprise $20 \%$ of the national cohort. BASHH/BHIVA guidelines recommend all patients have a sexual health assessment documented at presentation and 6monthly, with a full SHS offer annually and outcome documented. Aims We audited practice against these guidelines, to establish any difference in services offered to over-50s. We also examined rate of STI detection.

Methods 80 case notes were retrospectively audited-40 aged over 50 and 40 aged under 50 .

Results Abstract P121 Table 1 Shows the demographics of the groups. Only $75 \%$ of over-50s had sexual history documented at presentation compared to $86 \%$ of under- 50 s. $45 \%$ of $>50$ s had SHS documented at presentation (Chlamydia, GC and Syphilis), with a further $15 \%$ having Syphilis test only. $77 \%$ of over-50s had full SHS at baseline. In 24 baseline SHS performed in the over-50s we found 8 STIs: 2 Syphilis, 1 HSV, 1 Hepatitis B, 1 Chlamydia and 3 Gonorrhoea. In the 34 SHS performed in under-50s we found 11 STIs: 1 HPV, 2 Syphilis, 2 HSV, 1 Hepatitis B, 3 Chlamydia and 2 GC. Sexual history documentation in 2010/11 was poor in both groups. In the $>50$ s, only $28 \%$ had a documented sexual history. In the under-50s, $35 \%$ had documented sexual history. SHS in 2010/11 was offered to $20 \%$ of over-50s with 1 declining and in $35 \%$ of under-50s with 2 declining. Positivity was low; 1 case of Syphilis in each group.

Abstract P121 Table 1 Demographics of HIV cohort

\begin{tabular}{lcc}
\hline & $\boldsymbol{< 5 0}$ years & $>\mathbf{5 0}$ years \\
\hline Mean age & $82 \%$ & $62 \%$ \\
Male gender & 69 & 36 \\
MSM & $40 \%$ & $35 \%$ \\
White ethnicity & $55 \%$ & $68 \%$ \\
\hline
\end{tabular}

Conclusion In our sample, fewer over-50s had a sexual history and SHS offer documented at presentation. Screening at presentation identified STIs in both groups. Few patients declined SHS, suggesting patients find it acceptable. There were more STIs in the under-50s group at presentation, correlating to national trends. However, we tested more young patients, possibly explaining this difference. STI rate in 2010/11 was low but didn't differ with age-it is important to perform a sexual history in all patients and offer SHS, so we are developing a proforma to prompt clinicians.

\section{P122 MANAGEMENT OF SEXUAL ASSAULT IN THE EAST KENT COMMUNITY}

doi:10.1136/sextrans-2012-050601c.122

M Abubakar, ${ }^{*}$ A Shakya, K Forey. Kent Community Health NHS Trust, Kent, UK

Background Kent community health NHS Trust provides sexual health services (SHS) in East Kent serving an approximate population of 750000 based in eight clinical sites. SHS see a significant number of sexual assault (SA) case referrals in this region as there is no designated Sexual Assault Referral Centre.

Aim This audit was conducted to determine if the management of SA cases in the services is in accordance to the standards outlined by
British Sexual Health and HIV(BASHH) SA auditable outcome measures.

Method Case notes coded as SA based on a local code audited from the period of October 2010 to October 2011. Data were collected from the local SA proforma and case note documentation which identified information regarding victim's and assailant's profile and documentation of essential historical elements of SA. Other auditable outcomes measured included injury documentation, HIV risk assessment and post exposure prophylaxis (PEP) offer, police referral for crime and forensic examination (FME), self harm and child protection assessment. Data regarding emergency contraception, prophylactic antibiotics and hepatitis vaccination offered, testing for STIs and referral to EKRL were also collected.

\section{Results}

\begin{tabular}{ll}
\hline Total no SA codes & $92: 7$ notes missing: total 85 cases \\
Source of referrals & Self: $59(69.4 \%)$ Other: $26(30.6 \%)$ \\
Time since SA & $54(63.5 \%)$ seen within 6 weeks \\
Assailant details & Known: $51(60.0 \%)$ Unknown: $34(40.0 \%)$ \\
& HIV risk assessment: $100 \%$ \\
& Vaginal: $68(81.9 \%) \mathrm{N}=83$ \\
& Oral: $11(12.9 \%) \mathrm{N}=85$ \\
& Anal: $13(15.3 \%) \mathrm{N}=85$ \\
& High risk: $13-2$ eligible for PEP. Offered \\
& but declined \\
Yes $19(22.3 \%)$ No $66(77.7 \%) 10(11.7 \%)$ had \\
Referral for FME & FME prior attendance \\
& None \\
Referral to A and E & Yes $6(7.0 \%)$ No $79(93.0 \%)$ \\
Emergency contraception & Yes $6(7.0 \%)$ No $79(93.0 \%)$ \\
Prophylaxis antibiotics for STDs & $59(69.4 \%)$ indicated $43(72.9 \%)$ vaccinated \\
Hepatitis B vaccination & Yes $64(75.3 \%)$ No $21(24.7 \%)$ \\
First visit test & Yes $33(38.8 \%)$ No $52(61.2 \%)$ \\
Repeat test & $18(94.7 \%)$ N=19 \\
Child protection assessment under 16 & $55(64.7 \%)$ offered $5(0.09 \%)$ declined \\
Referral to police & Yes $48(56.4 \%)$ No $37(43.6 \%)$ \\
Referral to EKR line & $59(69.4 \%)$ \\
Follow-ups offered & $40(47.0 \%)$ \\
Total follow-ups seen &
\end{tabular}

Conclusions This audit highlighted the importance of clear documentation of treatment and care offered and provided to victims of SA. Poor documentation of management is likely to underestimate the overall outcome of care offered, but when a treatment is indicated and initiated there is a good clear documentation. The local SA proforma is concise and user friendly for any trained and qualified healthcare individuals and covers all aspects of care as recommended by BASHH except for the self harm assessment which will be included upon revision. A close working partnership with local police, SHS and EKRL is essential to ensure the management of SA within the community is in accordance with national standards.

\section{P123 NON-MEDICAL PRESCRIBING BY NURSE PRACTITIONERS IN A WALK-IN SEXUAL HEALTH CLINIC}

doi:10.1136/sextrans-2012-050601c.123

\section{A Black, ${ }^{*}$ C Roberts, A Smith. Imperial College Healthcare NHS Trust, London, UK}

Background Non-medical prescribing legislation in the UK provides suitably qualified nurses with prescribing powers comparable with doctors. This facilitates autonomous practice and allows independent completion of care episodes. There is however limited evidence regarding the application and safety of nurse prescribing. Aim Investigate the application of non-medical prescribing in sexual health. 
Methods A cross-sectional study explored non-medical prescribing in a London based GUM department between 1 January 2010 and 30 June 2010. A retrospective review of randomly selected clinical notes was performed. This included 382 nurse prescriber led and 255 nonprescriber led GUM consultations. Prescribing frequency; range of medications and diagnoses; independent episode completion and prescribing safety were investigated.

Results Medication was dispensed in $52.9 \%(n=337)$ of consultations in the 637 combined episodes. A total of 427 diagnoses were identified that required 34 different medicinal products and 452 treatments to be dispensed in total by nurse practitioners. The management of sexual contacts accounted for $22.1 \% \quad(n=60)$ of treatments. A statistical difference in independent practice existed between practitioners $\left(\chi^{2}\right.$ test $\left.\mathrm{p}<0.001\right)$, with prescribers 15.52 times (CI 9.41 to 25.59 ) more likely to independently complete episodes of care. Safe appropriate prescribing was identified in $99.1 \%$ $(n=210)$ of cases. On two occasions a lack of documentation of concurrent medication or allergies made it difficult to assess safety. There were no serious errors in prescribing practice found.

Conclusions In our GUM clinic widespread use of prescribing skills was demonstrated. Nurses with prescribing skills were able to work more independently. Non-medical prescribing has been applied safely in accordance with clear treatment guidelines.

\section{P124 A MOTIVATIONAL INTERVIEWING (MI) INTERVENTION AIMED AT REDUCING SEXUAL RISK TAKING IN MEN WHO HAVE SEX WITH MEN (MSM): THE LAUNCH OF A PILOT SERVICE}

doi:10.1136/sextrans-2012-050601c.124

${ }^{1} S$ Y Yap. ${ }^{2} \mathrm{D}$ Traynor. ${ }^{1}$ Central and North West London Foundation Trust, London, UK; ${ }^{2}$ Chelsea and Westminster Hospital, London, UK

Background HIV is one of the fastest growing serious health conditions in the UK. HIV+ MSM have an increased burden of sexually transmitted infections (STIs). Effective interventions are needed to reduce the risk to MSM's health. MI is an evidence based goal-directed approach to behaviour change. There is some evidence for its use in risk reduction in sexual health. A clinic was set up to deliver an MI based intervention to MSM who engage in "high risk" sexual activity. The aim is to reduce the frequency of patients' unprotected sex, thereby reducing risk of transmission/acquisition of STIs.

Aims The aim of this paper is to review the level of need for a MI based risk reduction clinic. An audit of referral data to the MI clinic was compared with an audit of referrals to the general sexual health psychology clinic. The latter receives referrals from sexual health; a proportion of total referrals to this service include clients who engage in high risk sexual activity.

Methods The MI clinic was publicised by presenting the clinic at teaching and multi-disciplinary clinic meetings. Patient leaflets outlining the service were distributed across clinic rooms. A survey of staff was carried out to assess potential need for the service. A comparison audit of referral data to the MI clinic vs the general sexual health psychology clinic over a 6-month period was carried out.

Results A comparison of the average number of referrals per month to the general psychology service vs the MI clinic was carried out. The MI clinic averaged $30 \%$ more referrals per month than the general sexual health psychology service. A survey of referrers showed that $67 \%$ of staff who were aware of the MI service reported that they had patients who would benefit from the service.

Discussion These results highlight the need for a specific service to address sexual risk taking. Further research is required to investigate whether MI is an effective intervention in this area.

\section{P125 THE ASSOCIATION BETWEEN CONDOM PROFICIENCY, CONDOM PROBLEMS AND STI RISK AMONG SCOTTISH MSM}

doi:10.1136/sextrans-2012-050601c.125

${ }^{1}$ L Goodall, ${ }^{* 1} \mathrm{D}$ Clutterbuck, ${ }^{2} \mathrm{P}$ Flowers. ${ }^{1}$ Chalmers Sexual Health Centre, Edinburgh, UK; ${ }^{2}$ Glasgow Caledonian University, Glasgow, UK

Background The effectiveness of condoms in preventing sexually transmitted infections (STI) including HIV depends on consistent and correct use.

Aims To examine associations between demographics, STI risk, condom proficiency, condom problems and STI acquisition among MSM and to direct discussion and debate towards thinking about how and why it might be important to improve condom use skills. Methods Cross-sectional surveys of MSM were conducted in GUM clinics and commercial gay venues in Summer 2010. The selfcompleted, anonymous questionnaires recorded data on socio-demographic variables, numbers of unprotected anal intercourse (UAI) partners in the preceding year, STIs diagnosed over the previous year and self-reported condom problems and condom proficiency.

Results 792 respondents provided data with an overall response rate of $70 \%$ ( $n=459$ clinic sample, $n=333$ community). Number of UAI partners was the strongest predictor of self-reported STI acquisition over the previous 12 months. Demographic characteristics were not associated with self-reported STI diagnosis. However, condom proficiency score was associated with self-reported STI acquisition in the previous 12 months. Condom problem score was also associated with self-reported STI diagnosis in the clinic but not community sample. Condom problem score remained associated with STI diagnosis after adjusting for number of UAI partners with logistic regression.

Discussion This study identified a measure of condom use associated with likelihood of STI diagnosis when controlling for number of UAI partners. Targeting those who experience condom problems may improve overall frequency and consistency of condom use among MSM; in turn reducing likelihood of STI acquisition. This could involve developing condom problem scales into screening tools for STI risk. Accordingly we encourage further research to determine the value of condom use training as a potential intervention to improve sexual health among MSM.

\section{P126 IMPROVING THE MANAGEMENT OF ANTENATAL WOMEN WITH POSITIVE SYPHILIS SEROLOGY WITHIN A GENITOURINARY MEDICINE SERVICE}

doi:10.1136/sextrans-2012-050601c.126

S Patel, ${ }^{*}$ R Aroney, M Bird, P Rice, P Hay. St. George's Hospital, London, UK

Background Congenital syphilis (STS) can be prevented through antenatal screening and treatment. The true incidence of congenital STS is unknown; $\sim 10$ cases/yr have been reported to the HPA by GUM clinics (probably reflects $30 \%-50 \%$ of cases). An audit at our centre ( $\sim 5000$ deliveries/yr) in 2004, highlighted suboptimal management of pregnant women with positive STS serology.

Methods A pathway has subsequently been developed and we report our findings since June 2004. Antenatal screening results are reviewed by a multidisciplinary team (MDT: virology, GU, antenatal, paediatric) experienced in the management of syphilis. Our GU team manages all pregnant women with positive serology who undergo full evaluation (incl. an STI check), further tests or treatment as necessary and follow-up of the neonate.

Results 123 referrals (108 pregnant women with positive STS serology) were received by the GU department from June 2004 to December 2009. Of these referrals, 36\% (44) had STS (1 primary, 1 secondary, 6 early latent, 36 late latent), 37\% (46) were previously 